\title{
Cultura, topoclimatología y cambios de clima en la zona andina del desierto de Atacama
}

\author{
Hugo Iván Romero Aravena ${ }^{1}$, Guillermo Espinoza ${ }^{2}$, Dustyn Opazo $^{1}$ y Daniela Sepúlveda ${ }^{1}$
}

1 Departamento de Geografía, Facultad de Arquitectura y Urbanismo, Universidad de Chile. hromero@uchilefau.cl

2 Centro de Estudios de Humedales, Centro de EstudiosdelDesarrollo. gespinoza @ ced.cl

Eixo: Climatologia em diferentes níveis escalares: mudanças e variabilidades

\begin{abstract}
Resumen
Lasinvestigaciones geográficas sobre cambios de clima deben considerar simultáneamentelasaproximacionestopoclimáticas estadísticas convencionales y aquellasculturales, basadasenlasprácticas, conocimientos y símbolos de las comunidades locales, para dar cuenta de lassíntesisterritoriales sobre las que lasociedadnecesita decidir sus estrategias de adaptación. Nuevos conceptos relativos aladíada clima-cultura permiten interpretar laepistemología, ontología y metodología que caracterizan a las redes de actores y sus topologíasespaciales, representadas en zonas topoclimáticas que caracterizan al Norte de Chile. Los topoclimas y loscambios de climas son revisitados a partir de interpretaciones etnográficas, actualizadasconinformaciones aportadas por talleres y entrevistas realizados enlas comunidades andinas de laregión de Tarapacá.
\end{abstract}

Palabras claves: topoclimatología, comunidades andinas, Norte de Chile

\section{Introducción}

Durante los últimos años y debido al creciente conocimiento sobre los cambios globales del clima, ha aumentado la sensación de riesgo en territorios cuya variabilidad espacio-temporal climática es muy alta como en el desierto de Atacama, uno de los paisajes más áridos del mundo. Para los pueblos originarios que han habitado por milenios estos aparentemente inhóspitos paisajes, la variabilidad y cambios de clima han sido parte sustancial de su existencia y permanencia (Nuñez et al., 2010), lo que implica conocimientos, prácticas, gestión e interpretación de sus territorios que requieren ser conocidos y comprendidos. Los desafíos que enfrenta la topoclimatología geográfica de estos lugares se define como una expresión sintética de las relaciones estrechas establecidas entre los componentes de los sistemas naturales y de éstos con la sociedad (Popke, 2016; Hulme, 2015) originando un híbrido que puede corresponder en la actualidad a una topoclimatología cultural o socioclimatología de los pueblos andinos (Romero y Mendonça, 2016; Romero y Opazo, 2016). 
XVII Simpósio Brasileiro

de Geografia Fisica Aplicada

I Congresso Nacional

de Geografia Física
OS DESAFIOS DA GEOGRAFIA FÍSICA NA FRONTEIRA DO CONHECIMENTO

Instituto de Geociências - Unicamp

Campinas - SP

28 de Junho à 02 de Julho de 2017

\section{Topoclimatología andina}

Se ha interpretado un conjunto de imágenes satelitales que proporcionan los patrones de distribución de las temperaturas superficiales y de productividad vegetal, de lo que resulta una compleja matriz comandada en primer lugar, por ejes latitudinales de influencia marítima, particularmente en la sección norte del área de estudio (fig.1), que posteriormente, avanzando hacia el sur, dan paso al predominio de la pampa árida y franjas longitudinales, destacando las cordilleras y altiplanos, que pueden ser alcanzados por el monzón amazónico y sus lluvias estivales (Romero et al., 2011 y 2013; Mendonça et. al, 2014). Por ello, el altiplano andino posee un extaordinario valor geográfico y territorial, debido a que las montañas que lo rodean localizan "torres de agua" cuyos recursos constituyen las únicas fuentes de que depende la vida. El clima de las culturas andinas es un híbrido socionatural, en la medida que significa no solo un evento stmosférico sino que también un constructo social que ordena fases de la vida cotidiana de las comunidades, en un sistema de conocimientos, prácticas y representaciones que no separa los hechos biofísicos de los metafísicos, los humanos de los no-humanos o la vida del tiempo y del espacio (Boelens, 2014; Romero y Mendonca, 2016).

SegúnMatheret al., (1980:285) latopoclimatología es "larelación sinérgica entre procesos climáticos, rasgos de lasuperficie terrestre y acciones humanas", cuyoestudiodebe unir a los geógrafos físicos, culturales y humanos para abordar una entidad híbrida, caracterizada por materialidades biofísicas y medioambientales, por un lado, y por conocimientossocioculturales, afectos y prácticas, por elotro (Popke, 2016:2). El desafíoes dar cuenta, valorizar y legitimar, diversos rangos de conocimientos, prácticas y experiencias, sobre loscuáleslas comunidades adoptandecisiones. La epistemología y ontología híbridas implican repensar lamaterialidaddel clima, reconociendo que haycomplejas redes de relaciones, comunalidades, interdependencias y comunicaciones entre lo humano y lo no humano, que conformanmanifestaciones y respuestas a loscambiosambientales (Hulme, 1987). Las comunidades indígenas andinas elaboranelconocimiento climático e interpretansu significado social desde puntos de vista muy distintos a los de lacienciaoccidental (Van Kessel y Salas, 2002). Para lainterpretación de esta ontología, se requierennuevos instrumentos, tales como laTeoría de losActantes-Redes y laTopología Semiótica Material (Popke, 2016, Goldman et al., 2016). Enlo que respecta a lametodología híbrida, combina enfoques interdisciplinariosdesarrolladoscon métodos cuantitativos y cualitativos, que complementandatos derivados de instrumentos o modelos científicos, concomprensiones interpretativas o etnográficas, desplazándose desde formas simplistas de explicaciónhaciaun más incierto pero vívido "sentido de encuentro" entre los humanos, las cosas, plantas, animales, tecnologías (Head and Gibson, 2012: 705), reunidos enlosayllus o chacras.

El clima y la cultura existen como una díada, solamenteeninterrelación (Hulme, 2015) y elprimerollega a ser culturizado a través de lasinterpretaciones simbólicas, y mediante ellas, los 
XVII Simpósio Brasileiro de Geografia Fisica Aplicada

I Congresso Nacional de Geografia Física

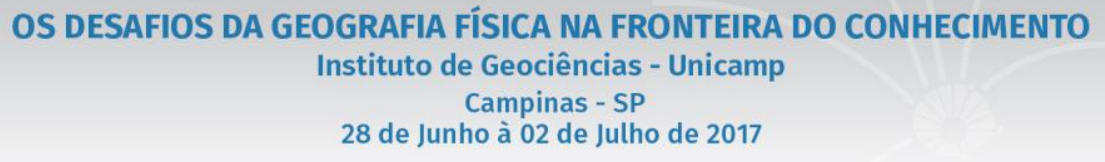

28 de Junho à 02 de Julho de 2017

humanos llegana estar aclimatados a lafisicalidaddeltiempo atmosférico. Esta interrelaciónacompañasiemprelasactuaciones de las comunidades andinas y tal como señalaTesuro (1988/1935) para el caso de las sociedades ancestrales japonesas, o Van Kessel y Salas (2012) para las andinas, el concepto separado de clima no existe enlas culturas locales. El clima y la cultura cambian constantemente y tanto laatmósfera como lasprácticas e imaginarioslocalesestánen constante flujoen escalas de tiempo y espacio diferentes. "La historia y el clima aislados uno de otroson meras abstracciones" (Tesuro, 1988; 1935:68).

Bajo esta concepción cultural del clima, cabríapreguntarse si es correcto aspirar a una estabilidad climática bajo control humano como objetivo político de bien público. Para Hulme (2008:14): "La idea de un clima global estable es una ilusiónenla medida que se trata de una co-construcción entre lafisicalidaddeltiempo atmosférico y lasideas y prácticas de las culturas humanas cuyasinterrelaciones entre estos mundos imaginativos y materiales cambiantes no son nunca estables" y difieren entre lugares. Para Hulme (2008), correspondería, por lo tanto, repensar laidea de cambio de clima, cuyas culturas conformansumaterialidad, y respecto a lascualeslapráctica de lacienciatiene lugar enmediosnaturales específicos con sus propios valores, supuestos y dinámicas de poder. La cultura no es menos central para lacomprensión e implementación de laadaptación al clima: laidentificación de losriesgos, lasdecisiones acerca de lasrespuestas, y losmedios de implementación, son mediados por la cultura, que es dinámica y reflexiva, y está a su vez conformada por laidea de cambio de clima. La cultura y suanálisis, soncentrales para comprenderlas causas y significados de loscambios de clima y susrespuestassociales, como unhecho cultural

\section{La topoclimatología estadística del Norte de Chile}

Sobre la base de un Sistema de Información Geográfica que incluyelas relaciones espaciales entre topografía, límites de cuencas, datos proporcionados por estaciones meteorológicas (cuadro 2) y ladistribución de la temperatura y vegetación, obtenida de imágenessatelitalescorrespondientes a losaños más húmedos y secos de la serie, se preparó una representación cartográfica (figura 3 a) y en forma paralela, se correlacionó, a través de un modelo de regresiónmúltiple, ladistribución espacial de las temperaturas medias de veranocon diferentes factores geográficos explicativos, tales como la altura, exposición, continentalidad y temperaturas de emisión superficial (Romero y Opazo, 2017), cuyarepresentación espacial se encuentraenla figura 3 b y suformulación estadística enelCuadro 1).

Cuadro N¹: Modelo de regresiónmúltiple 


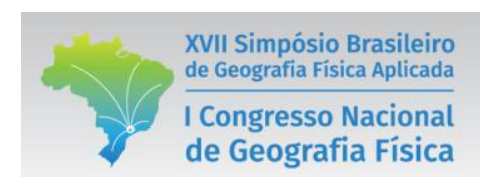
OS DESAFIOS DA GEOGRAFIA FÍSICA NA FRONTEIRA DO CONHECIMENTO Instituto de Geociências - Unicamp Campinas - SP
28 de Junho à 02 de Julho de 2017

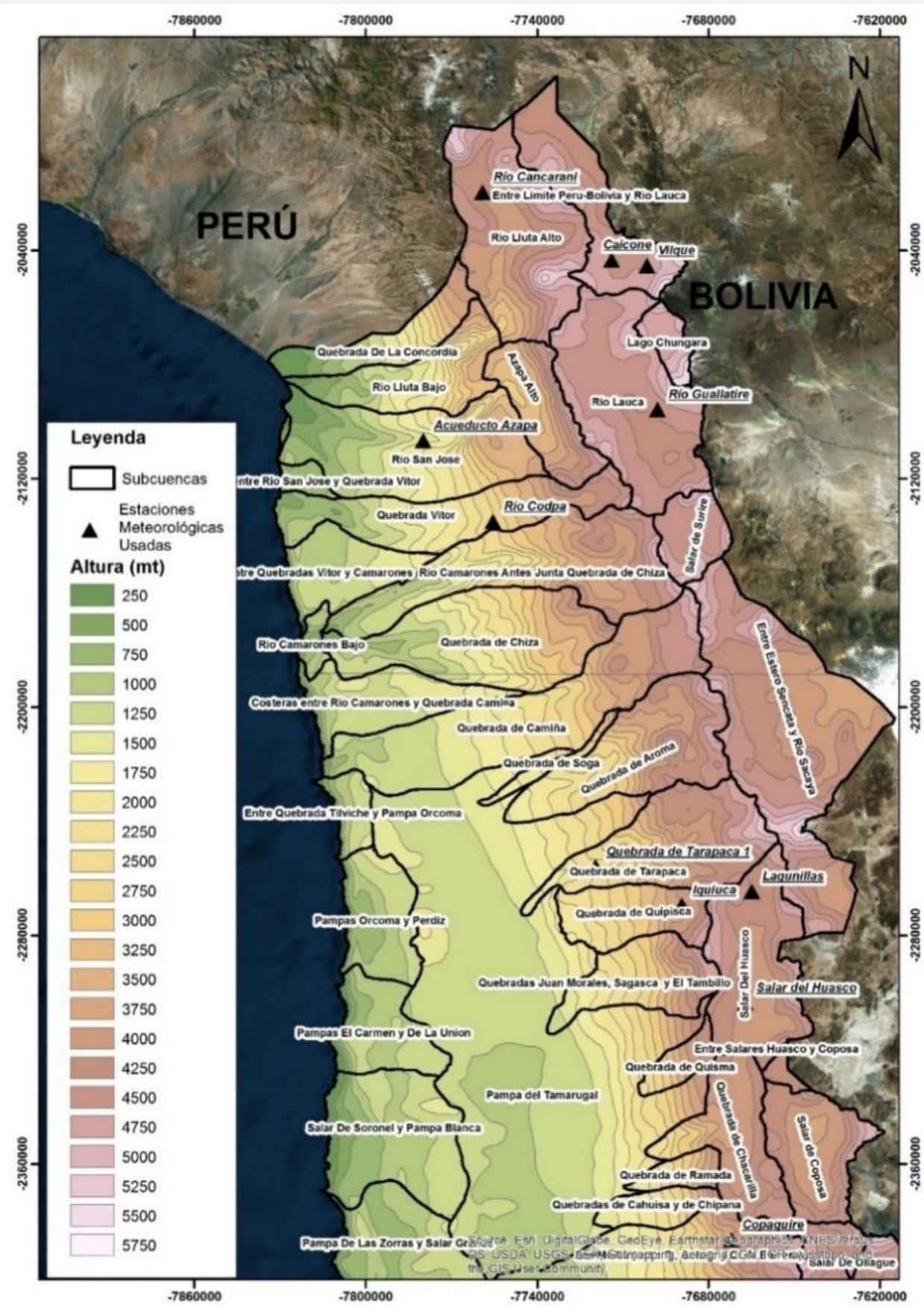

Figura $N^{\mathbf{0}}$ 1: Cuencas y subcuencasdel Norte de Chile y estaciones meteorológicas consideradas Cuadro No2: Temperatura promedio de verano, localización geográfica, distancia al mar, altura y exposición de las estaciones meteorológicas consideradas en el modelo de regresión múltiple 


\begin{tabular}{|c|c|c|c|c|c|c|}
\hline \multicolumn{2}{|c|}{$\begin{array}{l}\text { XVII Simpósio Brasileiro } \\
\text { de Geografia Fisica Aplicada } \\
\text { I Congresso Nacional } \\
\text { de Geografia Física }\end{array}$} & \multicolumn{5}{|c|}{$\begin{array}{l}\text { OS DESAFIOS DA GEOGRAFIA FÍSICA NA FRONTEIRA DO CONHECIMENTO } \\
\text { Instituto de Geociências - Unicamp } \\
\text { Campinas - SP } \\
28 \text { de Junho à } 02 \text { de Julho de } 2017\end{array}$} \\
\hline Estación Meteorológica & $\mid \begin{array}{c}\text { Temperatura promedio } \\
\text { de verano }\left({ }^{\circ} \mathrm{C}\right)\end{array}$ & $\begin{array}{c}\text { Distancia al mar } \\
(\mathrm{Km})\end{array}$ & Orientación & $\begin{array}{c}\text { Altura } \\
\text { (m.s.n.m) }\end{array}$ & Localización & Zona Topoclimática \\
\hline Acueducto Azapa & 13,67 & $50 \mathrm{~km}$ & Oeste & 1500 & N 7944399 E 406241 & $\begin{array}{l}\text { Zonas templadas de interfluvios de la zona } \\
\text { costera de las cuencas }\end{array}$ \\
\hline RïoCodpa & 14,62 & $76 \mathrm{~km}$ & Oeste & 1850 & N 7917546 E 421622 & Zonas templadas de transición sin vegetación \\
\hline Camiña & 12,3 & $86 \mathrm{~km}$ & Sur-oeste & 2500 & N 7864392 E 456168 & Zonas frías con vegetación de la precordillera \\
\hline Quebrada de Tarapacá & 10,2 & $87 \mathrm{~km}$ & Oeste & 2750 & N 7804366 E 463769 & $\begin{array}{l}\text { Zonas templadas de interfluvios de la zona } \\
\text { costera de las cuencas }\end{array}$ \\
\hline Río Caracarani & 6,81 & $96 \mathrm{~km}$ & Sur-oeste & 3908 & N 8027046 E 425885 & Zonas frías con vegetación de la precordillera \\
\hline Parca & 13,1 & $97 \mathrm{~km}$ & Oeste & 2500 & N 7787125 E 478731 & Zonas frías con vegetación de la precordillera \\
\hline Iquiuca & 9,3 & $118 \mathrm{~km}$ & Oeste & 3500 & N 7791887 E 492220 & Zonas frías con vegetación de la precordillera \\
\hline Caicone & 4,8 & $124 \mathrm{~km}$ & Nor-oeste & 4250 & N 8004258 E 468750 & Zonas con vegetación del Altiplano \\
\hline Río Guallatire & 5,8 & $128 \mathrm{~km}$ & Sur & 4280 & N 7955232 E 484223 & Zonas con vegetación del Altiplano \\
\hline Copaquire & 5,7 & $136 \mathrm{~km}$ & Oeste & 3750 & N 7682766 E 508490 & Zonas con vegetación del Altiplano \\
\hline Vilque & 5,3 & $136 \mathrm{~km}$ & Oeste & 4500 & N 8002389 E 480533 & Zonas con vegetación del Altiplano \\
\hline Huasco & 8,1 & $140 \mathrm{~km}$ & Sur-oeste & 4000 & N 7759885 E 513160 & Zonas frías de depresiones cordilleranas \\
\hline Lagunillas & 6 & $143 \mathrm{~km}$ & Sur-este & 4250 & N 7796412 E 515201 & Zonas con vegetación del Altiplano \\
\hline
\end{tabular}

El cuadro 3 permite observar que en general, las temperaturas registradas superan significativamente (sobre $2^{\circ} \mathrm{C}$ ) a los valores estimados en las estaciones Acueducto Azapa y Río Codpa, y sobre $1^{\circ} \mathrm{C}$ en Camiña y Parca. Las temperaturas estimadas superan a las registradas, aunque levemente, en las estaciones de Iquiuca, Vilque, salar de Huasco y Lagunillas, lo que se debería a condiciones topoclimáticas específicas de los sitios de registro. 

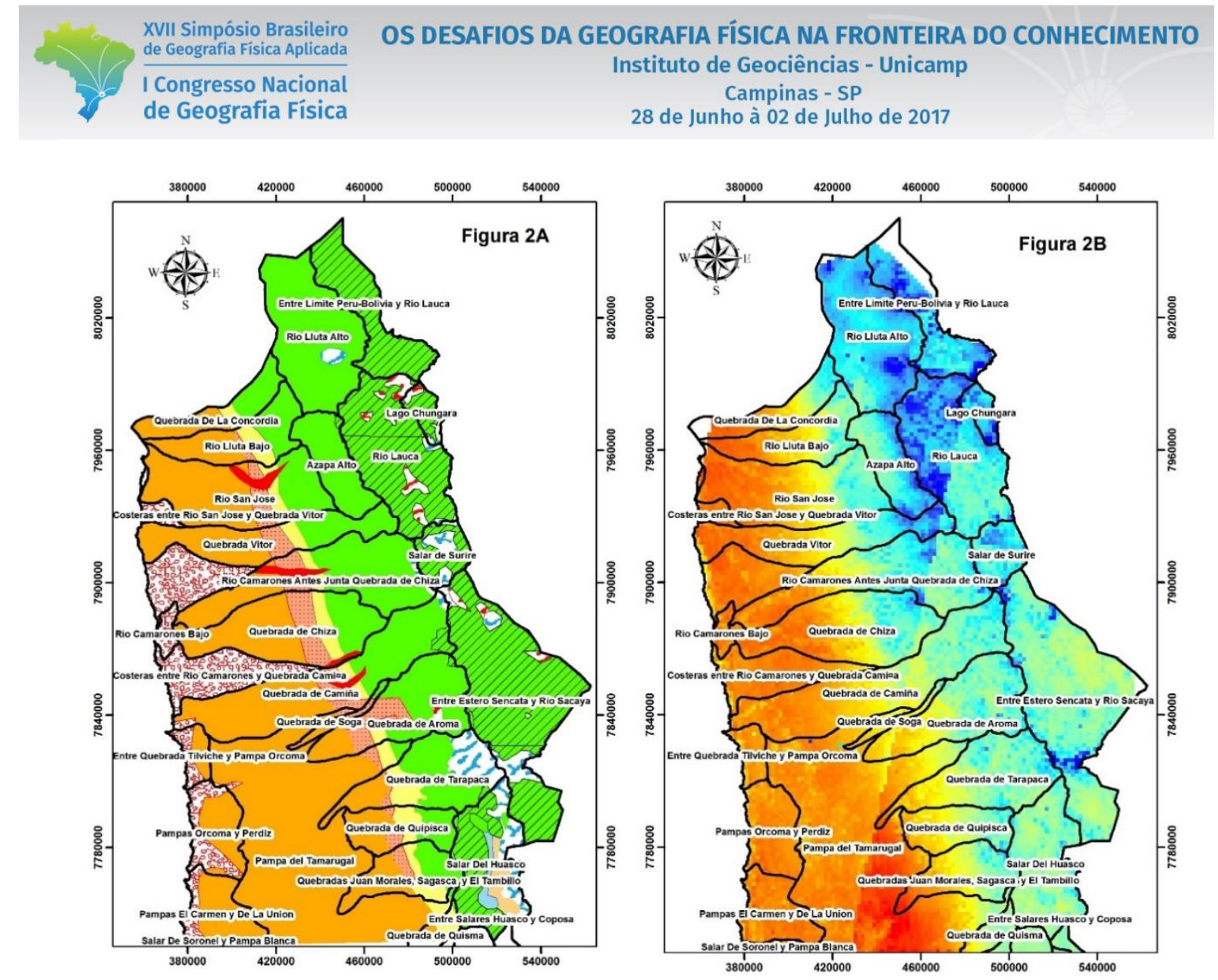

Topoclimas
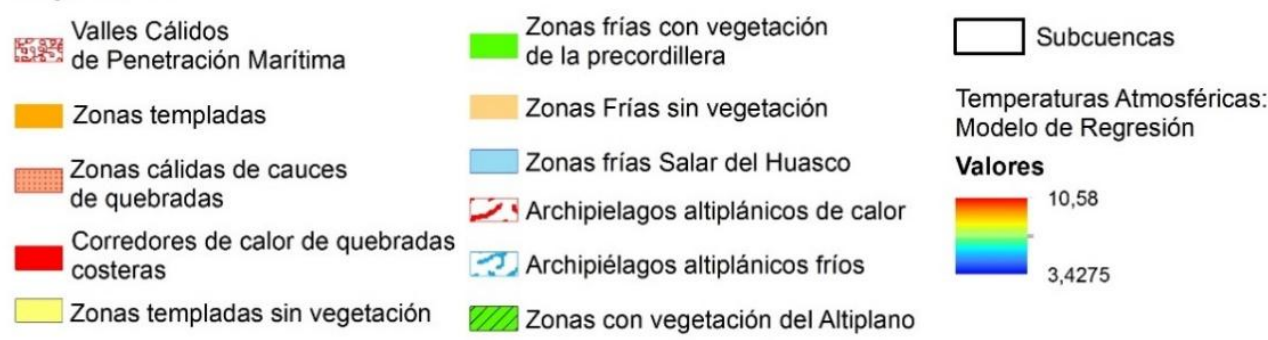

Figura N³: Zonas topoclimáticas interpretadas y estimadas en las regiones del Norte de Chile

\section{Climatología cultural de las comunidades andinas}

La climatología de lospueblos andinos se inscribeen una multiescalaridad que asocia funcional y simbólicamente no solo escalas propias de losespaciosregionales euclidianos sino que, preferentemente, redes ontológicas que vinculanlo real conlo simbólico, al dar cuenta de las grandes variabilidades espaciales y temporales que lacaracterizan. Susasentamientos se handesplazadohistóricamente sobre ladivisoria de aguas de laCordillera de los Andes, que separa las influencias áridas delocéano Pacífico de lasmasas de aire húmedas provenientes delMonzón Amazónico (Romero et al., 2011 y 2013; Mendonca et al., 2014) y enfunción de las diferentes cargas 

OS DESAFIOS DA GEOGRAFIA FÍSICA NA FRONTEIRA DO CONHECIMENTO Instituto de Geociências - Unicamp$$
\text { Campinas - SP }
$$
28 de Junho à 02 de Julho de 2017

de radiación, exposición a lasmasas de aire, grados de continentalidad y presencia de coberturas vegetales

Cuadro No3: Temperaturas promedio observadas y estimadas en las zonas topoclimáticas

\begin{tabular}{|c|c|c|}
\hline Estación & Temp. observada & Temp. estimada \\
\hline Acueducto Azapa & 13,67 & 11,1 \\
\hline RïoCodpa & 14,62 & 11,2 \\
\hline Camiña & 12,3 & 11,01 \\
\hline Quebrada de Tarapacá & 10,2 & 10,96 \\
\hline Río Caracarani & 6,81 & 6,29 \\
\hline Parca & 13,1 & 11,15 \\
\hline Iquiuca & 9,3 & 9,52 \\
\hline Caicone & 4,8 & 4,31 \\
\hline Río Guallatire & 5,8 & 5,33 \\
\hline Copaquire & 5,7 & 5,64 \\
\hline Vilque & 5,3 & 5,61 \\
\hline Huasco & 8,1 & 8,94 \\
\hline Lagunillas & 6 & 6,42 \\
\hline
\end{tabular}

Enlos casos analizados por Van Kessel y Salas (2002), Van Kessel (1992) y Grebe (1990), lainterpretación rítmica de los climas, identifica una estación de lluvias- lapara pacha- o de crianza de la vida, que abarca desde aproximadamente laprimeraquincena de noviembre hasta una similar en abril, cuando se registranprecipitaciones eventualmente torrenciales, truenos, fuertesvientos, granizos y nieve, que varían grandemente de lugar en lugar. A continuación, se desarrollalaestación de heladasgasapacha o chirawapacha, que abarca desde laprimeraquincena de abril a laprimera de agosto, donde loscielos despejados favorecenlaocurrencia de temperaturas muy bajas. Finalmente se registra laestación de secas- ch'akipacha- entre la segunda quincena de agosto y de noviembre, que alcanzasumayor aridez en este último mesdebido a laevaporación más elevada. La figura 4 es una ilustración modificada de la circularidad con que los aimaras de la región de Tarapacá diseñan icónicamente su calendario anual de actividades agrícolas y pastoriles, que constituyen la manera en que las variaciones climáticas son incorporadas en la vida cotidiana. Red de pastoreo (veranadas).

Conclusiones:

Los cambios de clima son estudiados, comprendidos y gestionados de manera diferente y muchas veces contradictoria por los enfoques empleados por la ciencia occidental y por los conocimientos 


\section{OS DESAFIOS DA GEOGRAFIA FÍSICA NA FRONTEIRA DO CONHECIMENTO Instituto de Geociências - Unicamp Campinas - SP \\ 28 de Junho à 02 de Julho de 2017}

locales de las comunidades indígenas andinas. Sus epistemologías, ontologías y metodologías son diferentes y por lo tanto, las actuaciones voluntarias o impuestas difícilmente van a conseguir acoplarse en medio de tales diferencias culturales, que resultan ser para las comunidades la parte fundamental de la diada tiempo atmosférica-cultura. La topoclimatología cultural puede contribuir a asociar diversas fuentes de conocimiento, proporcionando síntesis por las cuales los cambios de clima se aproximan mejor y a una escala más cercana a la vida cotidiana Mientras esto no se comprenda seguirán generándose conflictos, despojos e imposiciones que en nada contribuyen a la democracia y justicia ambiental de la zona. 
Figura 4: Calendario agrícola de las comunidades andinas de la región de Tarapacá, norte de Chile

\section{Bibliografia}

-BOELENS, R. (2014). Cultural policies and the hydrosocial cycle: Water, power and identity in the Andean highlands. Geofroum Volume 57, November 2014:234-247.

-GOLDMAN, M.; Daly, M. and Lovell, E. (2016). Exploring multiple ontologies of drought in agro-pastoral regions of Northern Tanzania: a topological approach.Area, 2016, 48.1:27-33.

-GREBE, M. E. (1990). Concepción del tiempo en la cultura aymara: representaciones icónicas, cognición y simbolismo. Revista Chilena de Antropología. Facultad de Ciencias Sociales, Universidad de Chile. (9:63-81).

-HEAD, L. and GIBSON, C. (2012). Becoming differently modern: geographic contributions to agenerative climate politics. Progress in Human Geography 37:93-114.

-HULME, M. (2015). Climate and its changes: a cultural appraisal. Geo: Geography and Environment, published by John Wilwy and Sons.

-HULME, M. (2009). Cosmopolitan Climates: hybridity, foresight and meaning. Theory, Culture and Society on Global Heating: social theory looks at climate change. 
-HULME, M. (2007). Geographical work at the boundaries of climate change. Transactions of the Institute of British Geographers 33:5-11.

-MATHER, J.R.; FIELD, R.T.; KALLSTEIN, R.S. AND WILMOTT, C.J. (1980). Climatology: the challenges of the eighties. Professional Geography 32: 285-92.

-MENDONÇA, M., ROMERO, H. y OPAZO, D. (2014). Análise multiescalar para a comprenssao de causas e consequencias da variabilidade climática na América do Sul. Experimentos em Climatología Geográfica. Organizadores: Charlei Aparecido de Silva, Edson Soares Fialho e Ercilio Torres Steinke. AsociaciónBrasileña de Climatología Geográfica, pp. 271-290.

-NUÑEZ, L., GROSJEAN, M., and CARTAJENA, I. (2010), Sequentialanalysisofhumanoccupationpatternandresource use in the Atacama Desert. Chungará, Vol. 42, $\mathrm{N}^{\circ} 2$ : 363-391.

-POPKE, J. (2016). Researching the hybrid geographies of climate change:reflections from the field. Area, 2016, 48, 1: 2-6.

-ROMERO, H.; OPAZO, D .y SEPULVEDA, D. (2017). Topoclimatología andina del norte de Chile: estimaciones estadísticas e interpretaciones culturales. XVI Encuentro de Geógrafos Latinoamericanos, Universidad Mayor de San Andrés, La Paz, Bolivia, 24-28 de abril de 2017

-ROMERO, H. y OPAZO, D. (2016). Socioclimas, riesgos y ordenación del territorio en las comunidades altoandinas del desierto de Atacama, En Clima, Sociedad,Riesgos y Ordenación del Territorio. Jorge Olcina, Antonio Rico y Enrique Moltó (editorees), Asociación Española de Climatología Serie A, nº10: 610-612.

-ROMERO, H. y MENDONCA, M. (2016). Socioclimas y glocalización en el Desierto de Atacama. IX Simposio Latinoamericano y V Iberoamericano de Geografía Física. Guimaraes, Portugal.

-ROMERO, H., MENDONCA, M., MENDEZ, M. y SMITH, P. (2011). Multiescalaridad, relaciones espaciales y desafíos ecológico-sociales de laclimatologíasudamericana. El caso deldesierto de Atacama. Revista Brasileira de Climatologia.Año 7.Vol. 8. Pp.7-29.

-ROMERO, H., MENDONCA, M., MENDEZ, M. y SMITH, P. (2013) Macro y mesoclimasdel Altiplano Andino y Desierto de Atacama: Desafíos y estrategias de adaptación social ante suvariabilidad. Revista de Geografía Norte Grande, PUC-Chile, volumen 55, 2013. Pp. 19-41.

-ROMERO, H. y VINAGRE, J. (1985). Topoclimatología de la Cuenca delRíoMapocho. Inform. Geogr. Chile 32: 3-30.

-TESURO, W. (1988/1935). Climate and Culture: A philosophical study (Trans Bownas, G.) Green Wood Press, New York.

-VAN KESSEL, J. y SALAS, P.E. (2002). Señas y Señaleros de la Santa Tierra. Agronomía Andina. Número 4 de la Serie: "Wageningen studies on heterogeinity and relocalization" del Departamento de Sociología Rural de la Universidad de Wageningen, Holanda.

-VAN KESSEL, J. (1992). La organización tempo espacial del trabajo entre los aimaras de Tarapacá: la perspectiva mitológica En Etnicidad, economía y simbolismo en los Andes: II congreso. ARZE, Silvia (dir.). Nouvelle édition. Institut français d'études andines. 